\title{
Effectiveness of Bupivacaine Trigger Points Injections in the Treatment of Myofascial Pain Syndrome
}

${ }^{1}$ Rabiu Mohammed Bashir, ${ }^{2}$ Afeez Abdulrahman and ${ }^{3}$ Zakari Suleiman

Departments of Anaesthesia:

${ }^{1}$ Abubakar Tafawa Balewa Teaching Hospital, Bauchi, Nigeria

${ }^{2}$ Usmanu Danfodiyo University Teaching Hospital Sokoto, Nigeria

${ }^{3}$ University of Ilorin Teaching Hospital Ilorin, Ilorin Nigeria

Corresponding author:

Rabiu MB

Departments of Anaesthesia, Abubakar Tafawa Balewa

Teaching Hospital, Bauchi, Nigeria

E-mail: elbashir200@yahoo.com,

rabiu3754@gmail.com,

phone: 2348037049294

\begin{abstract}
:
Background: Myofascial pain syndrome is a regional or widespread muscular pain associated with tenderness localized to linear or nodular hardening, trigger points in one or group of muscles. It is a major cause of time loss from work and low quality of life. Injections of myofascial trigger points either with local anaesthetics, steroid, normal saline or without any agent have been proven to provide pain relief. This study aims to determine the effectiveness of trigger point injection with bupivacaine in treatment of myofascial pain syndrome and improvement in quality of life.
\end{abstract}

Methods: After Institutional Ethical Committee approval, forty one patients aged 16 years and above with myofacial pain syndrome had $0.5 \mathrm{mls}$ of $0.25 \%$ plain bupivacaine trigger points injections with size $26 \mathrm{G} 1 \frac{1}{2}$ inch hypodermic needle. Pain was assessed using Numerical Rating Scale and Pain disability questionnaire was administered to all the patients before the procedures and 1-month post-intervention (follow-up visits) to assessed functional and psychological component of pain disability scale and their response to treatment.

Results: The male to female ratio was 9:1, and the mean numeric pain scores before and at 1-month post-intervention were $7.24 \pm 1.0$ and $1.29 \pm 0.84 \quad(\mathrm{p}<0.05)$ respectively, indicating significant reduction in pain severity.

Conclusion: Bupivacaine trigger points injections is effective at reducing pain intensity and improved quality of life in patients with myofascial pain syndrome.

Keywords: Myofascial pain syndrome, trigger points injections, Quality of life

connective tissue surrounding and separating muscles (myofascial structures).

Trigger points are exquisitely tender area, which cause pain referred to a distant region, called the referred pain zone. Acute trauma or repetitive trauma may results to the excessive stress on muscle fibers and the formation of pain generator known as trigger points (2). Patients may develop a regional pain resulting in a decreased range of 
movement in the affected muscles or region. Trigger point may be present in one or group of muscles accompanied by pain, spasm, limitation of movement, trigger point can be active painful with or without movement or latent when trigger point is painful on palpation only $(3,4)$. The tender point seen in MFS is caused by abnormal stresses on the muscles from sudden stress on shortened muscle or skeletal asymmetry, abnormal posture for long period of time, anaemia, low level of some essential cations and vitamin B complex has been implicated, this deficiency may be contributory other condition such as fatigue, radiculopathy, hyperthyroidism.( s). MFS can occur in any part of the body $30-93 \%$, craniofacial $55 \%$ and $21 \%$ lumbo-gluteal (3-5). There is no specific test for MFS, however basic routine test such as urinalysis and pack cell volume may be done. Usually diagnosis is clinical, characterize by presence of altered sensation or pain on pressure on nodule along the area of expected distribution. Demonstration of spontaneous increase in activity on electromyography with characteristic of active foci in the nodule or band is pathognomic (4).

Evaluation of myofascial pain includes locating the trigger points and muscles involved by Palpation of a hypersensitive bundle or nodule of muscle fiber of harder than normal consistency is the physical finding typically associated with a trigger point. Palpation of the trigger point will elicit pain directly over the affected area and/or cause radiation of pain toward a zone of reference known as referred pain and a local twitch response, well as recognition of these contributing factors (1-3). Myofascial pain syndrome is frequently overlooked as a diagnosis because it is often accompanied by signs and symptoms in addition to pain, coincidental pathology conditions, and behavioural and psychosocial problems.

Management of myofascial pain is multidisciplinary approach which includes medication, physical therapy (trigger points injection, muscle exercises), clinical psychology, proper exercise, nutrition Management, splint therapy, and behavioural therapy that depends on complexity of the case (6). The use of Trigger Point injection in treatment of myofascial pain syndrome can be done as dry needling or by injecting parental formulation of various drugs such as local anaesthetic agents and steroids Dexamethasone has been used in various study but risk of myopathies associate with its use and lack of rapid pain relief which limited its clinical application. Local anaesthetic agent provide rapid pain relief and membrane stabilization of the affected muscle following trigger point injection, Lidocaine has also been studied however its short duration of action about $40 \mathrm{~min}$ to 1 hour resulted in need for the search of local anaesthetic agents with relatively longer duration of action $(1,5)$ Bupivacaine is a local anaesthetic agent that provide analgesia between 4-5 hours with resultant rapid pain relief and improving quality of life. The short-term goal is to restore the muscle tot to normal length, posture and full joint range of motion with bupivacaine trigger point therapy.

\section{Materials and Methods}

Institutional Ethic committee clearance was obtained, and informed consent obtained from patient. Patients successive patients aged $\geq 18$ years (37 male and 4 female) with active Trigger Points presented to a pain clinic in North western Nigeria. Detailed pre-procedural review was done including history of previous systemic disease, history of drug allergy and hypersensitivity to local anaesthetic agents and patients on analgesics for more than one week or co analgesics such as Gabapentine, Amitryptiline and patients that missed the follow up were excluded.

The Pain Disability Questionnaire (PDQ) and NRS were used to measure perceived pain and disability. The Pain Disability Questionnaire was administered during the 1st visit (pre-injection) and on 1 month (post-injection). The trigger points were identified as per the diagnostic criteria (at least two of the essential criteria), and Trigger Point Injections were given with all resuscitation drugs and equipment available. The procedure was done under aseptic technique area to be injected was prepared with methylated spirit, the trigger point was palpated within the taut muscle band \& stabilized between two fingers of the non-dominant hand. The needle was inserted through the skin and passed into the zone of trigger point, presence of local twitch or pain elicited directly over the affected area and/or cause radiation of pain toward a zone of reference known as referred pain when similar pain experienced by the patient is reproduce indicated correct placement of the needle into the trigger point. After aspiration with a syringe with 26 - gauge needle of $1 / 2$ inch or $24-\mathrm{G}$ of $1 \frac{1}{2}$-inch needle depending on the depth to reach the trigger point, all patients received standard dose of $0.5 \mathrm{ml}$ of $0.25 \%$ bupivacaine injected into the trigger point. Signs of local toxicity such as light headiness, circum oral numbness and dizziness were looked for 
during observation and patients were discharged home after for 2 hours.

The PDQ is a 15-item questionnaire used in assessing disability status related to pain which correlates with quality of life assessment (QOL) and Numerical Rating Score (NRS) is a simple pain assessment tool that measure the severity of pain from 0 no pain, 1-3 mild pain, 4-6 moderate pain, 7-9 severe pain and 10 worst unimaginable pain, was used at pre intervention and one month post intervention. The PDQ contains two factors, a functional status component \& a psychosocial component; the individual items attributed to each factor are added to generate the score for the two different components and the total scores was composed of all the responses added together. This measure has demonstrated solid psychometric properties, including validity, reliability, and responsiveness to change. Data collected were analyzed using SPSS (Statistical Package for Social Sciences) Version 25. The result of analysis was presented using tables and charts, descriptive analyses for continuous variables were presented as mean and standard deviation, while categorical variables were presented as frequency and percentages. The paired samples T-test procedure compares means of two variables that represent the same group at different time while level of statistical significance was set at $P \leq 0.05$. This study aims to determine the effectiveness of trigger point injection with bupivacaine in treatment of myofascial pain syndrome and improvement in quality of life.

\section{Results}

Fifty-four patients were recruited from May 2015 to April 2017 only forty one patients completed the follow up.

Table 1: Demographic variables

\begin{tabular}{lcl}
\hline Variables & Frequency $\mathbf{n}=\mathbf{4 1}$ & $\mathbf{\%}$ \\
\hline Age & 13 & \\
$10-20$ & 28 & 31.7 \\
$21-30$ & & 68.3 \\
Sex & 34 & \\
male & 7 & 90.2 \\
female & & 9.8 \\
Occupation & 10 & \\
Skills & 25 & 24 \\
Un skill & 6 & 61 \\
Un employ & & 14.6 \\
\hline
\end{tabular}

Table 1: Showed majority of the study participant were in their second- and third-decade life, with majority of them in the age range of 21-30. There is gender preponderance of myofascial pain syndrome, with Male account for $90.2 \%$ of the study participants, majority $61 \%$ of the patients were unskilled labourers.

\section{Table 3: Paired T test}

\begin{tabular}{lccc}
\hline Variables & \multicolumn{2}{c}{$95 \%$ CI } & $p$-value \\
& lower & upper & \\
\hline $\begin{array}{l}\text { Pre-intervention NRS- } \\
\text { post-intervention scores }\end{array}$ & $5.55 \pm 1.28$ & $6.36 \pm 1.28$ & 0.001 \\
$\begin{array}{l}\text { Pre-intervention PDFS- } \\
\text { Post-Intervention PDFS }\end{array}$ & $47.95 \pm 15$ & $55.62 \pm 12.15$ & 0.001 \\
$\begin{array}{l}\text { Pre-intervention PDSS- } \\
\text { Post-intervention PDSS }\end{array}$ & $28.03 \pm 8.42$ & $33.34 \pm 8.42$ & 0.001 \\
\hline
\end{tabular}

$\mathrm{CI}=$ Confidence interval, $\mathrm{NRS}=$ Numerical Rating Score, PDFS $=$ Pain Disability Functional Score $\mathrm{PDSS}=$ Pain Disability Psychological Score

Improvement in pain observed on numerical rating score between pre and post intervention was statistically significant $(p$-value $=0,001)$. The difference in preintervention functional and psychological scores component and post intervention was found to statistically significant $(p$-value $=0.001)$ as showed in table

Table 4: Correlation of response to needling with mean scores Numerical Rating, Functional and Psychological scores of pain disability scale.

\begin{tabular}{|c|c|c|c|c|c|c|}
\hline \multirow{2}{*}{$\begin{array}{l}\text { Response to } \\
\text { needling } \\
\text { Variables }\end{array}$} & \multicolumn{2}{|l|}{ NRS $( \pm$ SD) } & \multicolumn{2}{|c|}{ Functional $( \pm$ SD) } & \multicolumn{2}{|c|}{ Psychological ( \pm SD) } \\
\hline & $\begin{array}{l}\text { Pre- } \\
\text { intervention }\end{array}$ & $\begin{array}{l}\text { Post- } \\
\text { intervention }\end{array}$ & $\begin{array}{l}\text { Pre- } \\
\text { intervention }\end{array}$ & $\begin{array}{l}\text { Post- } \\
\text { intervention }\end{array}$ & $\begin{array}{l}\text { Pre- } \\
\text { intervention }\end{array}$ & $\begin{array}{l}\text { Post- } \\
\text { intervention }\end{array}$ \\
\hline $\begin{array}{l}\text { Referred pain } \\
\text { local twitch }\end{array}$ & $7.26 \pm 1.05$ & $1.22 \pm 0.7$ & $73.35 \pm 12.0$ & $20.04 \pm 5.05$ & $45.74 \pm 7.76$ & $13.83 \pm 3.90$ \\
\hline \multicolumn{7}{|l|}{ response } \\
\hline $\begin{array}{l}\text { Referred pain } \\
\text { only }\end{array}$ & $7.17 \pm 1.33$ & $1.33 \pm 0.8$ & $70.88 \pm 11.33$ & $18.62 \pm 4.7$ & $42.50 \pm 7.76$ & $15.00 \pm 4.7$ \\
\hline $\begin{array}{l}\text { Local twitch } \\
\text { response }\end{array}$ & $7.26 \pm 0.96$ & $1.00 \pm 1.0$ & $71.55 \pm 11.70$ & $21.25 \pm 1.71$ & $48.50 \pm 6.60$ & $14.25 \pm 5.32$ \\
\hline None & $7.25 \pm 0.71$ & $1.63 \pm 1.1$ & $71.33 \pm 10.91$ & $17.33 \pm 2.94$ & $43.33 \pm 7.23$ & $15.50 \pm 1.87$ \\
\hline Total & $7.24 \pm 0.99$ & $1.30 \pm 0.8$ & $71.26 \pm 11.62$ & $19.49 \pm 4.52$ & $45.02 \pm 7.53$ & $14.34 \pm 3.91$ \\
\hline
\end{tabular}

Table 4: Showed patients with Referred pain and local twitch response have higher pre intervention Numerical Rating and Functional Score of 7.26 \pm 1.05 and $73.35 \pm 12.0$ respectively, which is higher than the mean average scores among patients that had referred pain, local twitch response only and those with no response. 
Post intervention results revealed better pain regression among patients with local twitch only $1.00 \pm 1.0$ and higher improvement in functional 17.33 \pm 2.94 and psychological $13.83 \pm 3.90$ scores among patients with no response and referred pain with local twitch respectively, indicating better improvement in quality of life.

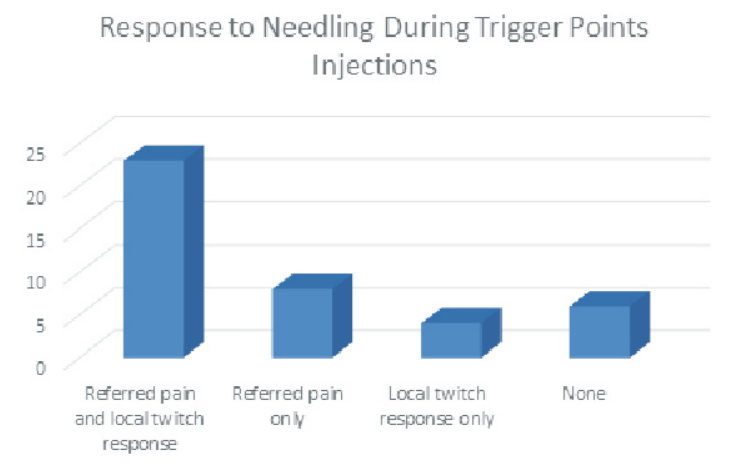

Figure 1: Showed 23 (56.1\%) of the patients had both local twitch and referred pain during trigger point injection while $8(19.5 \%)$ and $4(9.7 \%)$ had only twitch and referred pain respectively, $6(14.6 \%)$ of the patients had no referred pain or local twitch response.

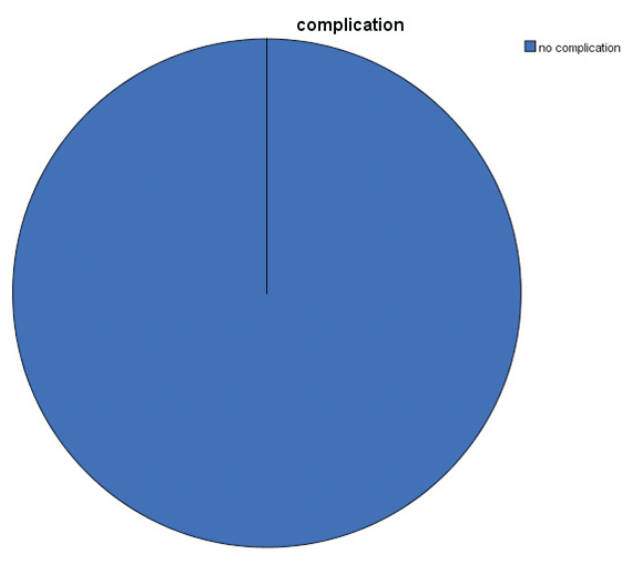

Figure 2: No complication was recorded during the procedure and follow up

\section{Discussion}

This study revealed significant reduction in pain intensity after Trigger point injection among the study population. The Pain Disability Scale (PDS) assesses the functional as well as the psychosocial component of pain was used in this study to assess quality of life (QOL). Our study showed significant reduction in both functional and psychological scores after trigger point injection was observed indicating an improvement in quality of life, after bupivacaine trigger point injection, reduction in pre intervention functional and psychological component of PDS in comparison with post trigger point injection functional and psychological scores on pain disability scale indicated effectiveness of trigger point injection with bupivacaine in improving quality of life at one month follow up.

Pain Disability Questionnaire (PDQ) was used in this study to correlated disability status related to pain which reflect quality of life, the PDQ which contains two factors, a functional status component and a psychosocial component, there was proportionate decrease in post intervention functional scores compared to psychological scores this may be due to the fact that addition of bupivacaine may provide immediate relief and deactivation of trigger zone and provide longer analgesia enough to restored muscle function and return to daily normal activities while the emotional experience will required longer time through reassurance to be restored. Therefore, even short-term relief may significantly in improve QOL which is essential for gaining patients confidence. Jafri et al (1) concluded in their review article that Myofascial pain is a significant health problem affecting as much as $85 \%$ of the general population and the onset and persistence of myofascial pain syndrome are known to be caused by myofascial trigger points. A mechanistic understanding of myofascial trigger points is critical to developing treatments for myofascial pain syndrome (7). Gerber et al (4) concluded that, long-term trigger point injection appears to be a well-tolerated adjunct treatment modality for various types of myofascial pain. The efficacy was demonstrated by a questionnaire answered by a smaller cohort. Further prospective study is suggested to attest this conclusion. Carel B et al (5) have conclusively proved in their study that the percentage of muscles having active and latent trigger points deactivated using trigger point injection was very high in non-traumatic shoulder pain. In a prospective study conducted by AY S et al (8) aimed to compare the efficacy of local anesthetic injection and dry needling methods on pain, cervical range of motion (ROM), and depression in myofascial pain syndrome patients (MPS). The authors reported statistically significant improvements in Visual analogue score (VAS), cervical Range of Movement (ROM) and Beck Depression Inventory (BDI) scores after 4 and 12 weeks in both groups compared to pre-treatment results $(p<0.05)$. Although in their study both patient groups 
were given stretching exercises aimed at the trapezius muscle to be applied at home while in our study no muscle exercise or any physiotherapy was used.

Clinically, trigger point can be located by Palpation of an area of extreme tenderness, harder than normal consistency; occasionally a bundle or nodule of muscle fiber may be felt. Hypersensitive on physical finding typically associated with a trigger point. Correct needle placement within the trigger point will elicit pain directly over the affected area and/or cause radiation of pain toward a zone of reference and a local twitch response (2$4)$.

In our study 23 (56.1\%) patients have local twitch with referred pain were found to have higher pre-intervention pain score $7.26 \pm 1.05$ but patient with twitch only 4 $(9.8 \%)$ during needling of trigger point showed better post-intervention pain regression $1.00 \pm 1.0$ indicating presence twitch response correlated with correct needle placement into the active trigger zone, which is essential for successful deactivation trigger point. Similar findings was reported by Hong CZ (9) in his study done to assessed the importance of twitch response during trigger point injection, the researcher concluded that it is essential to elicit local twitch response during injection to obtain an immediately desirable effect. In another singleblinded study conducted by $\mathrm{Ga} \mathrm{H}$ et al (10), pain scores, range of neck movement, pressure pain intensity and depression were measured up to four weeks from the first treatment. The authors reported that Local twitch responses were elicited at least once in $94.9 \%$ of all subjects and was significantly associated with reduction in pain score, improvement in range of movement and depression trend. In their study multiple injection was given this may results in higher twitch response observed compared to our study, we used single injection technique.

Presence of local twitch response does not correlate with changes in pain and disability, and multiple systematic reviews have failed to conclude whether the local twitch response is relevant to the outcome of trigger point injection (11). Post needling soreness is consistently reported in studies using repeated in and out needling to elicit local twitch response and increases in proportion to the number of needle insertions, in contrast needle winding without local twitch response to myofascial trigger points and connective tissue is well supported in the literature, as it is linked to anti-nociception and factors related to tissue repair and remodeling $(9,11)$, the positive biochemical changes in the trigger points after needling may simply be a wash out effect related to local vasodilation. While the local twitch response during trigger point injection may not unnecessary be associated with to better outcome for managing myofascial pain, further investigation is required.

Our study revealed 23 (56.1\%) patients had referred pain and local twitch response while only $8(19.5 \%)$ patients had referred pain, six $(14.6 \%)$ patients have no referred pain or local twitch response during trigger point injection. In this study, patients with neither referred pain nor local twitch response were found to have lower preintervention mean pain score with better postintervention pain remission compared to those with referred pain and local twitch response. Similarly, patients with no response during needling had better improvement in functional component of PDS indicating significant improvement in pain and quality of life irrespective of response during needling.

This study demonstrated that presence of referred pain and or local twitch response may be associated with pain intensity of the trigger point and necessary related to the outcome of trigger point injection. Presence of twitch or referred pain during trigger point injection with bupivacaine may be essential clinical diagnostic indicator for correct placement of needle within the trigger point which is necessary for successful deactivation of the pain generator (trigger point). Hong $\mathrm{CZ}$ et al (12), in their study to investigate the occurrence of referred pain elicited by needle injection during myofascial trigger point injection, and to assess the correlated factors, including the pain intensity of an active myofascial trigger point and the occurrence of local twitch response. The authors reported that both Palpation and Injection referred pain were elicited in $53.9 \%$ of the patients, during trigger point injection referred pain was elicited in $33.7 \%$ of the patients while $12.3 \%$ have no referred pain during trigger point injection.

The occurrence of referred pain and local twitch response was significantly correlated to the pain intensity of active myofascial trigger point, the authors concluded that frequency of occurrence of referred pain is mainly depends on pain intensity of an active myofascial trigger point. Although the authors didn't correlate the presence of referred pain to the success of trigger point injection 
Effectiveness of Bupivacaine Trigger Points Injections in the Treatment of Myofascial Pain Syndrome

$(11,12)$. None of patients had any complication during the procedure either none was reported after during follow up. The difficulty in managing myofascial pain syndrome lies in the critical need to match the multidisplinary management approach. Failure to address the entire problem through a team approach may lead to failure to resolve the primary cause of the pain which may progress to chronic pain syndrome.

\section{Conclusion}

This study demonstrated that, trigger Point Injections with bupivacaine in patients with myofascial pain syndrome was effective for pain regression and improvement in quality of life. However, the significance of this study lies in the fact that it is safe, minimally invasive procedure, with minimum or no side-effects. Long-term effectiveness of this procedure requires longer follow-up and a more detailed study on the affected patient population

\section{Conflict of Interest}

No conflict of interest declared by any of the authors

\section{Funding}

No fund was received for the purpose of this study.

\section{References}

1. Jafri SM. Review Article on Mechanisms of Myofascial Pain" International Scholarly Research Notices Volume 2014 (2014), Articleill 523924 16pages
2. Alvarez DJ,Rockwell PG.Trigger point:Diagnosis and management. Am Fam physician.2002 feb 15;65(4):653-60

3. Simons DG, Travell JG, Simons LS, Cummings BD. Travel \& Simons Myofascial Pain and Dysfunction: The Trigger Point Manual Lippincott Williams \& Wilkins, 1998

4. Gerber LH, Sikdar S, Armstrong K et al. A Systematic comparison between subjects with no pain and pain associated with active myofascial trigger points" PM\& R: The Journal of Injury. Function and Rehabilitation. vol. 5, no. 11, pp. 931.-938 2013.

5. Carel B, Jan D. High prevalence of shoulder girdle muscles with myofascial trigger points in patients with shoulder pain", BMC Musculoskeletal Disorders, 2011,12:139, doi:10.1186/1471-24 7 412-139.

6. Esenyel M Caglar N, Aldemir T. Treatment of myofascial pain AM J Phys Med Rehabil.2000 Jan Feb;79(1):48-52.

7. Frictious JR, Steenks MH. Diagnosis and treatment of myofascial pain. Ned Tijdschr Tandheelkd.1996 July;103(7):249-53

8. AYS, Evlik D, Tur BS. Comparison of injection methods in myofascial pain syndrome: A randomized control trial. Clin Rheumatol.2010 Jan 29(1):19-23

9. Hong CZ. Lidocaine injection versus dry needling to myfascial trigger point.The importance of twitch response. Amj phys med Rehabil 1994 July-Aug;73(3):256-63

10. Ga H, Choi JH, Park CH, Yoon HJ.Acupuncture versus Lidocaine injectionof Trigger point in elderly patients.A Randomized trial. Acupun med 2007 Dec;25 (4):130-6

11. Perreaunault T, Dunning J, Butts $R$. The Twitch response during trigger point dry needling: Is it necessary for successful outcome.

12. Hong CZ, Kuan TS,Chen JT,Chen SM. Referred pain elicited by palpation and by needling of myofascial trigger point: A comparison. Arch Phys Med Rehabil.1997 Sep;78(9):957-60 